\title{
Comparison of nutrient composition in kernel of tenera and clonal materials of oil palm (Elaeis guineensis Jacq.).
}

\begin{abstract}
Nutritive information about oil palm kernel is scarce, especially on the composition of sugars and water-soluble vitamins. This study aims to evaluate both tenera and clonal materials for their proximate composition, fatty acid profile, amino acid composition, sugar, mineral and water-soluble vitamin contents. The tenera material had a higher moisture, fat and fibre content as compared to the clonal material, whereas protein, carbohydrate and ash content were higher in the clonal material. The major fatty acid constituents in palm kernel oil were lauric acid, myristic acid and oleic acid. The palm kernel proteins were deficient in lysine and tryptophan but rich in glutamic acid, arginine and aspartic acid. Sucrose was the most abundant sugar in palm kernel. The mineral analysis of the samples showed high levels of potassium, phosphorus, magnesium, calcium and manganese, while niacin was the watersoluble vitamin present at the highest concentrations in palm kernel.
\end{abstract}

Keyword: Oil palm (Elaeis guineensis) kernel; Palm kernel oil; Nutrient composition; Mineral; Fatty acid; Sugar; Vitamin; Amino acid; Protein. 